\title{
Leucoplasias bucais: relação clínico-histopatológica
}

\section{Oral leukoplakias: clinical-histopathologic relation}

\author{
Tânia Lemos Coelho RODRIGUES* \\ Lino João da COSTA** \\ Maria Carmeli Correia SAMPAIO** \\ Fabiano Gonzaga RODRIGUES*** \\ Antônio de Lisboa Lopes COSTA****
}

\begin{abstract}
RODRIGUES, T. L. C.; COSTA, L. J. da; SAMPAIO, M. C. C.; RODRIGUES, F. G.; COSTA, A. de L. L. Leucoplasias bucais: relação clínico-histopatológica. Pesqui Odontol Bras, v. 14, n. 4, p. 357-361, out./dez. 2000.

A relação entre o aspecto clínico e as características histológicas das leucoplasias bucais foi avaliada em 28 pacientes adultos, que apresentaram lesões leucoplásicas na mucosa bucal. As lesões foram divididas quanto ao aspecto clínico em homogêneas e não-homogêneas, e classificadas segundo suas características histopatológicas em seis grupos: hiperceratose com ausência de displasia epitelial; displasia epitelial leve; displasia epitelial moderada; displasia epitelial severa, carcinoma in situ e carcinoma invasivo. Os resultados clínicos mostraram maior ocorrência de leucoplasias homogêneas $(78,6 \%)$ do que não-homogêneas $(21,4 \%)$. Os achados histopatológicos demonstraram que $32,2 \%$ dos casos apresentaram hiperceratose com ausência de displasia epitelial, 53,5\% evidenciaram displasia epitelial (39,3\% leve, $7,1 \%$ moderada e $7,1 \%$ severa) e 14,3\% diagnosticados como carcinoma invasivo. As leucoplasias homogêneas apresentaram alterações celulares discretas, enquanto as não-homogêneas evidenciaram displasia epitelial severa e carcinoma invasivo. Os resultados sugerem um maior cuidado no diagnóstico e controle de leucoplasias dada a possibilidade de transformação maligna.
\end{abstract}

UNITERMOS: Leucoplasia bucal; Neoplasias bucais.

\section{INTRODUÇÃO}

O termo "leucoplasia" foi utilizado pela primeira vez, segundo GRINSPAN ${ }^{10}$ (1973), por SCHWIMMER, em 1877, para caracterizar lesões brancas da mucosa bucal sem causa definida. Desde então, esta denominação foi ampla e irrestritamente adotada, causando controvérsias. Em 1978, a Organização Mundial de Saúde (OMS) ${ }^{22}$ definiu-a como uma mancha ou placa branca da mucosa bucal, não removivel à raspagem, que não pode ser caracterizada clínica ou patologicamente como outra enfermidade. Atualmente, o termo "leucoplasia" é utilizado apenas no sentido "clínico" para denominar uma placa predominantemente branca da mucosa bucal, não removivel à raspagem, cuja superficie pode apresentar-se lisa, rugosa ou verrucosa ${ }^{2,15}$. Há de se salientar, entretanto, que este termo não traz qualquer conotação quanto às alterações histopatológicas.

A leucoplasia é a lesão cancerizável mais freqüente da cavidade bucal, desenvolvendo-se em qualquer região; no entanto, a mucosa jugal, o lábio inferior e a lingua têm sido as áreas mais afetadas $^{8}$. Sua etiologia está relacionada, em muitos casos, a hábitos como tabagismo, e outras vezes é considerada idiopática. Sua ocorrência se dá principalmente em pacientes de meia idade, do sexo masculino. Entretanto, SILVERMAN et al. ${ }^{17}$ (1976) ressaltaram que este fato provavelmente decorre da associação com o tabagismo, praticado com maior freqüência pelos homens, do que propriamente uma diferença sexual biológica.

O processo de diagnóstico da leucoplasia é um tanto complexo, não só pela diversidade de seu aspecto clínico, mas também pela ausência de sintomatologia, sendo geralmente descoberta em exames de rotina. Por isso, torna-se necessário fazer um diagnóstico por exclusão de outras lesões que se apresentam como placas brancas na mucosa bucal $^{7}$.

O quadro histológico da doença também apresenta variações; porém, duas características são consideradas importantes: a hiperceratose e a dis-

\footnotetext{
* Mestre em Estomatologia; ** Professores Doutores da Disciplina de Diagnóstico Bucal; *** Professor Assistente da Disciplina de Cirurgia Buco-Maxilo-Facial - Universidade Federal da Paraíba.

**** Professor Doutor da Disciplina de Patologia Oral da Universidade Federal do Rio Grande do Norte.
} 
RODRIGUES, T. L. C.; COSTA, L. J. da; SAMPAIO, M. C. C.; RODRIGUES, F. G.; COSTA, A. de L. L. Leucoplasias bucais: relação clínico-histopatológica. Pesqui Odontol Bras, v. 14, n. 4, p. 357-361, out./dez. 2000.

plasia epitelial em vários graus de severidade. Estas alterações são significativas do ponto de vista evolutivo da lesão, por determinarem comportamentos biológicos diferentes.

Considerando-se portanto, que a leucoplasia bucal é uma lesão cancerizável, ou seja, com potencial de sofrer transformação maligna, estudamos seus aspectos clínicos e microscópicos, procurando relacioná-los.

\section{MATERIAL E MÉTODO}

Nossa amostra foi composta de 28 pacientes adultos, de ambos os sexos, com idade acima de 35 anos, que ao exame clínico apresentavam leucoplasias bucais. Os mesmos foram selecionados no Ambulatório do Serviço de Cabeça e Pescoço do Hospital Napoleão Laureano (João Pessoa - PB), sendo submetidos a anamnese e exame clínico das lesões pelo mesmo cirurgião-dentista calibrado para esta função.

Como parte do processo de diagnóstico, as leucoplasias foram divididas clinicamente em dois tipos: homogênea e não-homogênea, baseando-se na classificação preconizada por AXÉLL et al. ${ }^{2}$ (1996), a qual define:

- leucoplasia homogênea: lesão predominantemente branca, de superficie plana, fina, que pode exibir fendas superficiais com aspecto liso, enrugado, ou corrugado, e textura consistente;

- leucoplasia não-homogênea: lesão predominantemente branca ou branco-avermelhada, que pode ter superficie irregular, nodular, ou exofitica.

Todas as lesões foram submetidas à biópsia excisional, em razão das mesmas serem únicas e de reduzido tamanho, não propiciando mapeamento e utilização de marcador com azul de toluidina. O material foi processado laboratorialmente e corado pela técnica de hematoxilina-eosina (H. E.). O exame histopatológico foi realizado por um único patologista calibrado para esta função.

Histologicamente, as lesões foram classificadas em seis grupos: hiperceratose com ausência de displasia epitelial; displasia epitelial leve; displasia epitelial moderada; displasia epitelial severa, carcinoma in situ e carcinoma invasivo.

A classificação dos casos de hiperceratose foi feita com base na espessura da camada de ceratina, ou seja, quando a mesma excedia a normalmente encontrada na área considerada.

A classificação dos casos de displasia epitelial foi feita com base no critério proposto por BÁNÓCZY; CSIBA ${ }^{4}$ (1976), que utiliza como parâ- metros histológicos: estratificação epitelial irregular, hiperplasia da camada basal, processos reticulares em forma de gota, aumento do número de figuras mitóticas, perda da polaridade das células basais, aumento da razão núcleo-citoplasma, polimorfismo nuclear, hipercromatismo nuclear, aumento do tamanho dos nucléolos, ceratinização de células isoladas ou em grupos na camada celular espinhosa e redução da aderência intercelular. Assim, os casos de displasia podem ser caracterizados como:

- displasia leve: quando duas características citadas anteriormente estão presentes;

- displasia moderada: quando duas a quatro das características citadas estão presentes;

- displasia severa: quando cinco ou mais das características citadas estão presentes.

Para a classificação dos casos em carcinoma in situ, foi necessário que toda a espessura do epitélio apresentasse as características celulares citadas anteriormente sem invasão do tecido conjuntivo subjacente.

Para os casos de carcinoma invasivo, foi necessário que as células exibissem todas as características citadas anteriormente, e estivessem arranjadas em lençóis, ninhos, cordões e isoladamente invadindo o tecido conjuntivo subjacente ${ }^{15}$.

\section{RESULTADOS E DISCUSSÃO}

Na classificação quanto ao aspecto clínico, obtivemos maior ocorrência de leucoplasias homogêneas $(78,6 \%)$ do que não-homogêneas $(21,4 \%)$. Estes dados estão em consonância com os resultados encontrados por vários autores como AXÉLL ${ }^{1}$ (1987), GUPTA et al. ${ }^{11}$ (1989) e SCHEPMAN et al. ${ }^{16}$ (1998), os quais consideraram a aparência clínica da variante homogênea como clássica e também mais freqüente. Este tipo clínico apresenta superficie plana, fina, com aspecto liso ou corrugado, e tem sido considerado menos agressivo do que o tipo não-homogêneo, que, por sua vez, é representado por lesões predominantemente brancas ou branco-avermelhadas de superficie irregular, nodular ou exofitica. Esta diferença não está apenas no aspecto clínico, visto que as lesões não-homogêneas, microscopicamente, estão mais freqüentemente associadas à ocorrência de displasia epitelial e, portanto, mais sujeitas a sofrerem malignização ${ }^{2,5,9,13,14}$.

Considerando-se o aspecto histopatológico, nossos resultados mostraram que $32,2 \%$ dos casos apresentaram hiperceratose com ausência de displasia epitelial. Dos casos com displasia epitelial, observamos a seguinte distribuição: 39,3\% - displasia 
RODRIGUES, T. L. C.; COSTA, L. J. da; SAMPAIO, M. C. C.; RODRIGUES, F. G.; COSTA, A. de L. L. Leucoplasias bucais: relação clínico-histopatológica. Pesqui Odontol Bras, v. 14, n. 4, p. 357-361, out./dez. 2000.

leve, $7,1 \%$ - displasia moderada e $7,1 \%$ - displasia severa. O restante, ou seja, 14,3\% foram diagnosticados como carcinoma invasivo, não sendo evidenciado nenhum caso de carcinoma in situ na nossa amostra. Portanto, 53,5\% das lesões pesquisadas apresentaram displasia epitelial, dados que estão em consonância com VEDTOFTE et al..$^{20}$ (1987) que constataram em sua investigação $57 \%$ de casos com displasia epitelial (Figuras 1, 2, 3, 4 e 5).

A presença de displasia epitelial representa em termos evolutivos um quadro intermediário entre a hiperceratose sem displasia e o carcinoma in situ ou carcinoma invasivo. Assim, devemos enfatizar que a ocorrência dessa alteração é um dado importante na avaliação do prognóstico da leucoplasia, pois casos com hiperceratose e displasia epitelial apresentam comportamentos biológicos diferentes.

A malignização geralmente ocorre em casos de

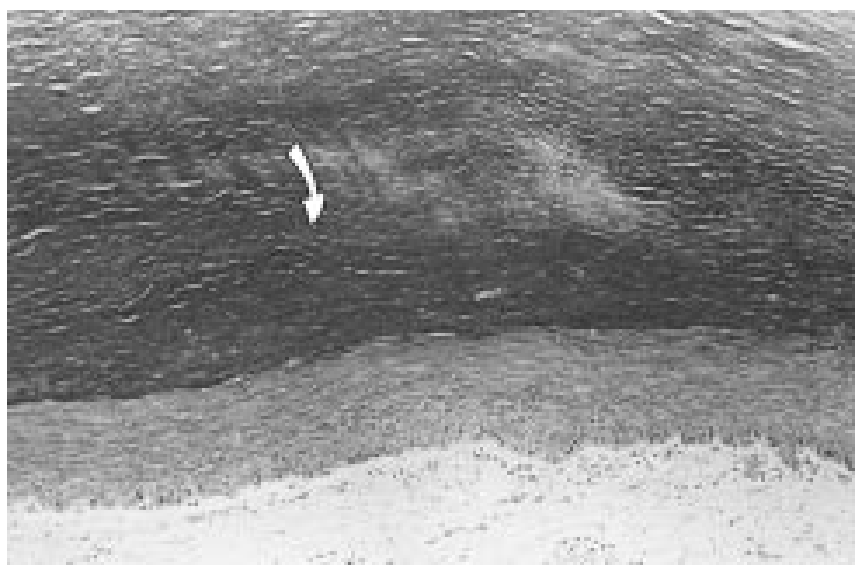

FIGURA 1 - Hiperceratose. Detalhe ressaltando a hiperceratinização (H. E., 100 X).

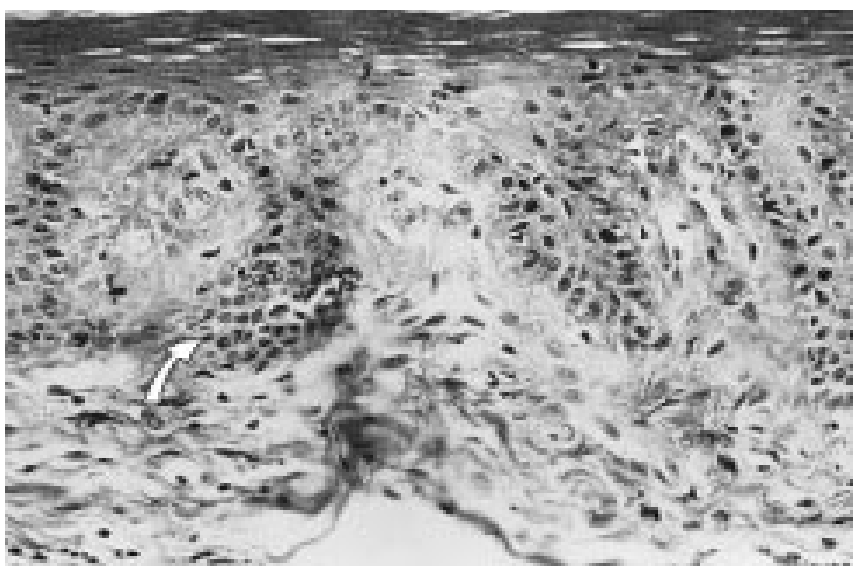

FIGURA 2 - Displasia epitelial leve. Detalhe demonstrando a projeção epitelial em forma de gota e hiperplasia basilar (H. E., $200 \mathrm{X})$. leucoplasias com displasia epitelial, ao contrário das lesões que evidenciam apenas hiperceratose, onde se observa um caráter exclusivamente benigno ${ }^{3,12,17,18}$. VAN DER WAAL et al. ${ }^{19}$ (1997) salientaram que apesar da displasia epitelial ser considerada como um importante fator preditivo de transformação maligna, nem todas as lesões com esta característica sofrerão o mesmo processo e, por outro lado, nem todas as lesões com ausência de displasia epitelial estão livres de se malignizarem. Neste contexto, recomendamos que as leucoplasias sejam não só biopsiadas, mas também acompanhadas periodicamente, buscando-se prevenir eventuais mudanças no seu comportamento clínico e histológico.

Visando correlacionar o aspecto clínico destas lesões e seu grau de diferenciação celular, observamos que dos 22 casos de lesões do tipo homogêneo, 9 apresentaram hiperceratose com ausência

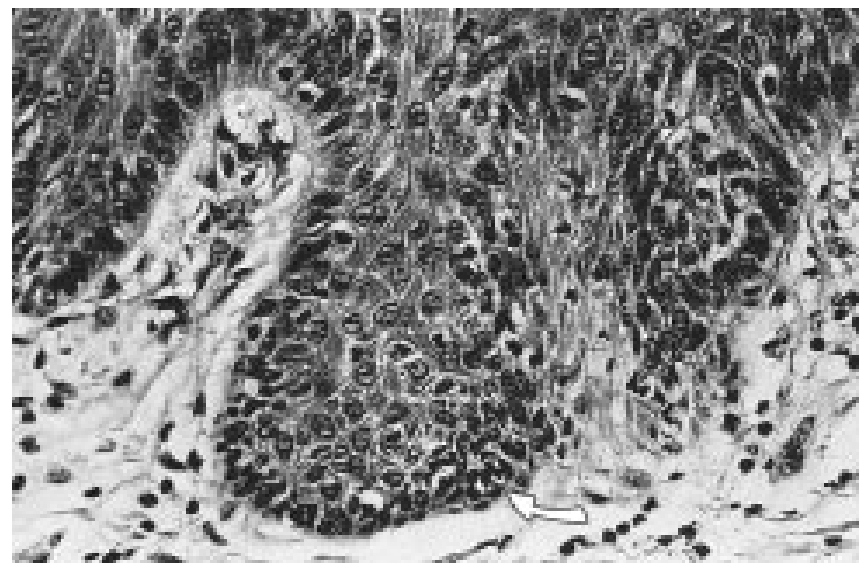

FIGURA 3 - Displasia epitelial moderada. Detalhe evidenciando a perda de adesão entre as células e pleomorfismo celular (H. E., $400 \mathrm{X}$ ).

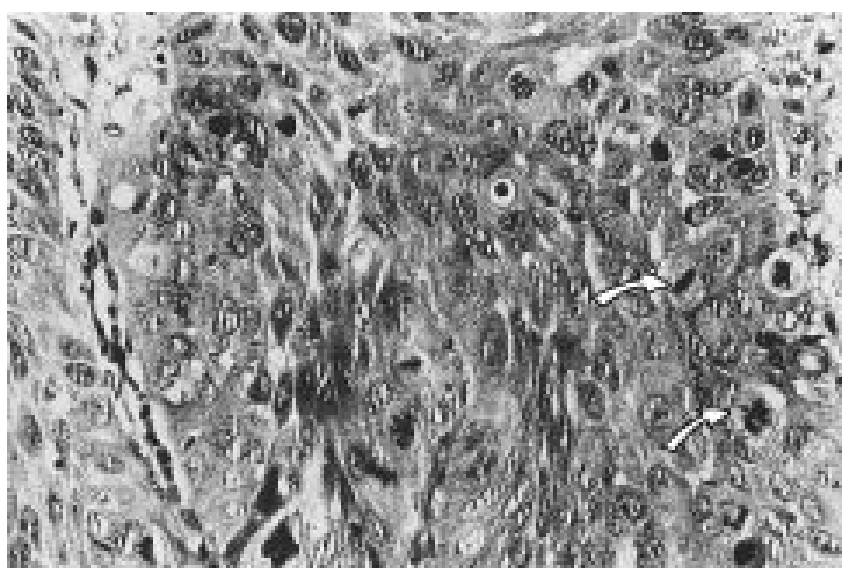

FIGURA 4 - Displasia epitelial severa. Detalhe ressaltando as mitoses atípicas em todas as camadas do epitélio e estratificação epitelial irregular (H. E., 400 X). 
RODRIGUES, T. L. C.; COSTA, L. J. da; SAMPAIO, M. C. C.; RODRIGUES, F. G.; COSTA, A. de L. L. Leucoplasias bucais: relação clínico-histopatológica. Pesqui Odontol Bras, v. 14, n. 4, p. 357-361, out./dez. 2000.

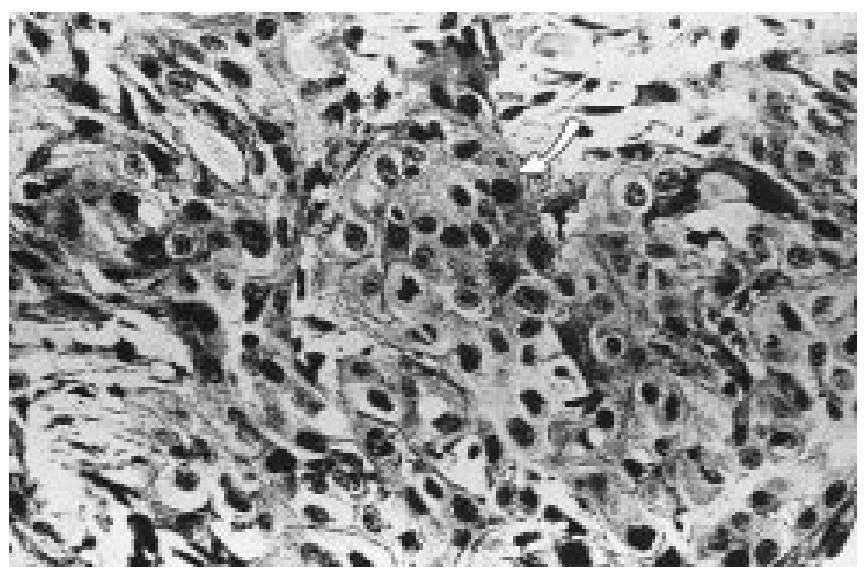

FIGURA 5 - Carcinoma invasivo. Detalhe evidenciando células neoplásicas arranjadas em ninhos sólidos que invadem o tecido conjuntivo subjacente (H. E., $400 \mathrm{X})$.

de displasia epitelial, 11 evidenciaram displasia leve e 2 displasia moderada. As leucoplasias homogêneas, no entanto, apresentaram em sua maioria um quadro histopatológico de displasia epitelial leve. Por outro lado, as leucoplasias não-homogêneas apresentaram alterações histológicas mais severas. Dos 6 casos de lesões não-homogêneas, 2 revelaram displasia epitelial severa e 4 evidenciaram carcinoma invasivo (Gráfico 1).

Nossos resultados demonstraram que existe uma relação entre o aspecto clínico da lesão e seu grau de diferenciação celular, pois as leucoplasias homogêneas tiveram quadros histológicos menos severos, caracterizados principalmente por displasia epitelial leve, enquanto os encontrados nas lesões não-homogêneas foram fundamentalmente de grau severo ou propriamente maligno. O que nos chama a atenção é que apesar de as leucoplasias poderem apresentar uma aparência clínica inócua, microscopicamente podem revelar características de malignidade.

Dos 4 casos $(14,3 \%)$ com diagnóstico de carcinoma invasivo, todos caracterizaram-se clinicamente como leucoplasias não-homogêneas, o que sugere que esta forma clínica apresenta maior propensão à transformação maligna. Estes dados são semelhantes aos observados por BOUQUOT ${ }^{6}$ (1986) em pesquisa com 1.787 pacientes portadores de leucoplasias, nos quais $12,2 \%$ das lesões revelaram-se como carcinoma espinocelular. Mais recentemente, SCHEPMAN et al. ${ }^{16}$ (1998) em sua investigação verificaram também uma taxa de $12 \%$.

Levando-se em consideração estes resultados, e diante da diversidade de aspectos clínicos e histopatológicos apresentados pelas leucoplasias bucais, torna-se obrigatório biopsiá-las, pois apenas

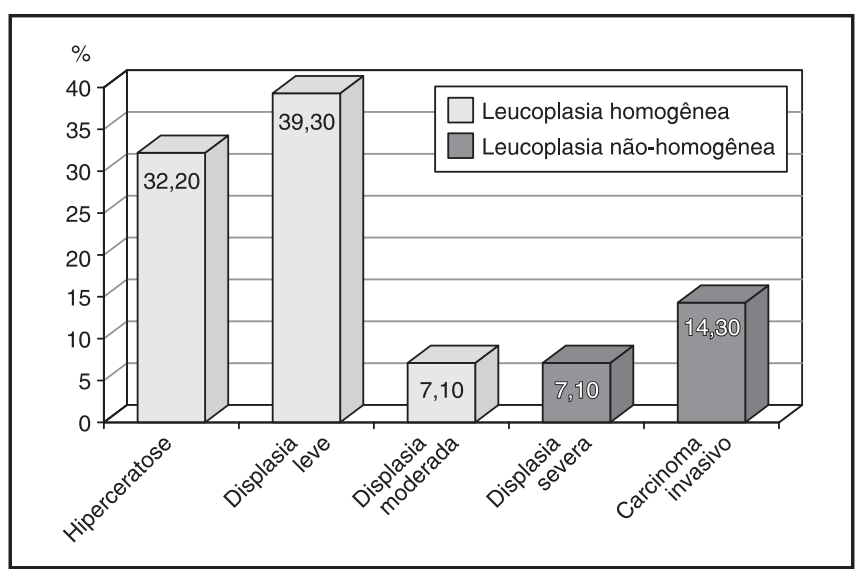

GRÁFICO 1 - Distribuição das leucoplasias bucais segundo a relação clínico-histopatológica.

por meio do exame histopatológico obtém-se seu diagnóstico definitivo ${ }^{3,7,18,19,21}$.

Além da relação entre o aspecto clínico das leucoplasias bucais e suas características histopatológicas, outros dados como localização anatômica, idade e hábitos nocivos dos portadores também devem ser cuidadosamente avaliados na elaboração do diagnóstico e prognóstico destas lesões.

Assim, o cirurgião-dentista deve estar sempre preparado para detectar esta patologia por meio do exame clínico, bem como ser capaz de avaliar possiveis fatores relacionados, pois esta é uma lesão cancerizável. Neste contexto, o profissional poderá contribuir para o diagnóstico precoce do câncer bucal e, por conseguinte, para o sucesso no seu tratamento.

\section{CONCLUSÃO}

Em função dos resultados obtidos, concluímos que existe uma relação entre o aspecto clínico e as alterações histopatológicas das leucoplasias bucais. As leucoplasias homogêneas apresentaram alterações celulares discretas, enquanto as nãohomogêneas evidenciaram características histopatológicas de displasia epitelial severa ou mesmo de carcinoma invasivo. Nossos resultados sugerem um maior cuidado no diagnóstico e controle das leucoplasias dada a possibilidade de transformação maligna.

\section{AGRADECIMENTOS}

À direção do Hospital Napoleão Laureano e ao Dr. Ary Serrano Santos por permitirem a seleção dos pacientes no Ambulatório do Serviço de Cabeça e Pescoço, e à técnica de laboratório Nemilzia Alves de Moura pela realização da parte histotécnica desta pesquisa. 
RODRIGUES, T. L. C.; COSTA, L. J. da; SAMPAIO, M. C. C.; RODRIGUES, F. G.; COSTA, A. de L. L. Leucoplasias bucais: relação clínico-histopatológica. Pesqui Odontol Bras, v. 14, n. 4, p. 357-361, out./dez. 2000.

RODRIGUES, T. L. C.; COSTA, L. J. da; SAMPAIO, M. C. C.; RODRIGUES, F. G.; COSTA, A. de L. L. Oral leukoplakias: clinical-histophathologic relation. Pesqui Odontol Bras, v. 14, n. 4, p. 357-361, out./dez. 2000.

The relation between the clinical aspects and histologic characteristics of oral leukoplakias was evaluated on 28 adult patients presenting with oral leukoplakia. The lesions were clinically classified as homogeneous and nonhomogeneous, and divided regarding their histopathologic characteristics in six groups: hyperkeratosis without epithelial dysplasia; mild epithelial dysplasia; moderate epithelial dysplasia; severe epithelial dysplasia, squamous cell carcinoma in situ and invasive carcinoma. Homogeneous leukoplakia comprised $78.6 \%$ of the cases and nonhomogeneous, $21.4 \%$. Microscopically, $32.2 \%$ of the lesions disclosed hyperkeratosis without evidences of epithelial dysplasia, $53.5 \%$ epithelial dysplasia (39.3\% mild, $7.1 \%$ moderate, and $7.1 \%$ severe) and $14.3 \%$ invasive carcinoma. Homogeneous leukoplakia showed discreet histologic changes, while nonhomogeneous lesions evidenced histologic characteristics of severe epithelial dysplasia and even of invasive carcinoma. The results suggest great precaution in the diagnosis and follow-up of leukoplakia, considering the possibility of malignant transformation.

UNITERMS: Leukoplakia, oral; Mouth neoplasms.

\section{REFERÊNCIAS BIBLIOGRÁFICAS}

1. AXÉLL, T. Occurence of leukoplakia and some other oral white lesions among 20,333 adult Swedish people. Community Dent Oral Epidemiol, v. 15, n. 1, p. 46-51, Feb. 1987

2. AXÉLL, T.; PINDBORG, J. J.; SMITH, C. J.; van der WALL, I. Oral white lesions with special reference to precancerous and tobacco-related lesions: conclusions of an international symposium held in Uppsala, Sweden, May 18-21, 1994. J Oral Pathol Med, v. 25, n. 2, p. 49-54, Feb. 1996

3. BÁNÓCZY, J. Follow-up studies in oral leukoplakia. J Maxillofac Surg, v. 5, n. 1, p. 69-75, Feb. 1977.

4. BÁNÓCZY, J. C.; SIBA, A. Occurence of epithelial dysplasia in oral leukoplakia. Oral Surg Oral Med Oral Pathol, v. 42, n. 6, p. 766-774, Dec. 1976.

5. BÁNÓCZY, J.; SÚGAR, L. Longitudinal studies in oral leukoplakia. J Oral Pathol, v. 1, n. 6, p. 265-272, 1972.

6. BOUQUOT, J. E. Common oral lesions found during a mass screening examination. J Am Dent Assoc, v. 112, n. 1, p. 50-57, Jan. 1986.

7. __ Reviewing oral leukoplakia - clinical concepts for the 1990's. J Am Dent Assoc, v. 122, n. 7, p. 80-82, June 1991.

8. BOUQUOT, J. E.; GORLIN, R. J. Leukoplakia, lichen planus and other oral keratoses in 23,616 white Americans over the age of 35 years. Oral Surg Oral Med Oral Pathol, v. 61, n. 4, p. 373-381, Apr. 1986.

9. BOUQUOT, J. E.; WHITAKER, S. B. Oral leukoplakia - rationale for diagnosis and prognosis of its clinical subtypes or "phases". Quintessence Int, v. 25, n. 4, p. 133-140, Feb. 1994

10. GRINSPAN, D. Enfermidades de la boca, Tomo II, Patología. Clínica y terapéutica de la mucosa bucal, Mundi, Buenos Aires, 1973.

11. GUPTA, P. C.; BHONSLE, R. B.; MURTI, P. R. et al. An epidemiologic assessment of cancer risk in oral precancerous lesions in India with special reference to nodular leukoplakia. Cancer, v. 63, n. 1, p. 2247-2252, June 1989.
12. LIND, P. O. Malignant transformation in oral leukoplakia. Scand J Dent Res, v. 95, n. 6, p. 449-455, Dec. 1987.

13. PINDBORG, J. J.; RENSTRUP, G.; POULSEN, H. E. et al. Studies in oral leukoplakias. V. Clinical and histological signs of malignancy. Acta Odontol Scand, v. 21, n. 3, p. $407-414,1963$.

14. PINDBORG, J. J.; JOLST, O.; RENSTRUP, G. et al. Studies in oral leukoplakia: a preliminary report on the period prevalence of malignant transformation in leukoplakia based on a follow-up study of 248 patients. J Am Dent Assoc, v. 76, n. 4, p. 767-771, Apr. 1968.

15. PINDBORG, J. J.; REICHART, P. A.; SMITH, C. J. et al. WHO: Histological typing of cancer and precancer of the oral mucosa, 2. ed. New York : Springer, 1997, $87 \mathrm{p}$.

16. SCHEPMAN, K. P.; van der MEIJ, E. H.; SMEELE, L. E. et al. Malignant transformation of oral leukoplakia: a follow-up study of a hospital-based population of 166 patients with oral leukoplakia from the Netherlands. Oral Oncol, v. 34, n. 4, p. 270-275, July 1998.

17. SILVERMAN Jr., S.; BHARGAVA, R.; MANI, N. J. et al. Malignant transformation and natural history of oral leukoplakia in 57,518 industrial workers in Gujurat, India. Cancer, v. 38, n. 4, p. 1790-1795, Oct. 1976.

18. SILVERMAN, S. J.; GORSKY, M.; LOZADA, F. Oral leukoplakia and malignant transformation. Cancer, v. 53, n. 1, p. 563-568, Feb. 1984.

19. van der WAAL, I.; SCHEPMAN, K. P.; van der MEIJ, E. H., et al. Oral leukoplakia: a clinicopathological review. Oral Oncol, v. 33, n. 5, p. 291-301, Oct. 1997.

20. VEDTOFTE, P.; HOLMSTRUP, P.; HJORTING-HANSEN, E. et al. Surgical treatment of premalignant lesions of oral mucosa. Int $\mathbf{J}$ Oral Maxillofac Surg, v. 16, n. 6, p. 656-664, Dec. 1987.

21. WALDRON, C. A.; SHAFER, W. G. Leukoplakia revisited: a clinicopathologic study of 3,256 oral leukoplakias. Cancer, v. 36, n. 4, p. 1386-1392, Oct. 1975.

22. WHO. Colaboration Centre for Oral Precancerous Lesions. Definition of leukoplakia and related lesions: an aid to studies on oral precancer. Oral Surg Oral Med Oral Pathol, v. 46, n. 4, p. 518-539, Oct. 1978. 